\title{
Fatty Acid Binding Protein-4 Silencing Attenuates Atherosclerosis Progression by Affecting Macrophage Apoptosis and Autophagy
}

\author{
Siqin Zhaorigetu' ${ }^{1}$, Priyanka Prathipati², Cristian Rodriguez-Aguayo ${ }^{3,4}$, \\ Brian L. Walton ${ }^{2}$, Anil K. Sood $4,5,6$, Gabriel Berestein-Lopez ${ }^{3,4,5}$
}

\begin{abstract}
${ }^{1}$ Department of Pediatric Surgery, Center for Advanced Heart Failure, University of Texas Health Science Center at Houston, Houston, TX, USA

${ }^{2}$ Department of Advanced Cardiopulmonary Therapies and Transplantation, Center for Advanced Heart Failure, University of Texas Health Science Center at Houston, Houston, TX, USA

${ }^{3}$ Department of Experimental Therapeutics, The University of Texas M.D. Anderson Cancer Center, Houston, TX, USA

${ }^{4}$ The Center for RNA Interference and Non-Coding RNA, The University of Texas M.D. Anderson Cancer Center, Houston, TX, USA

${ }^{5}$ Department of Cancer Biology, The University of Texas M.D. Anderson Cancer Center, Houston, TX, USA

${ }^{6}$ Department of Gynecology Oncology \& Reproductive Medicine, The University of Texas M.D. Anderson Cancer Center, Houston, TX, USA
\end{abstract}

Email: ^Brian.Walton@uth.tmc.edu

How to cite this paper: Zhaorigetu, S., Prathipati, P., Rodriguez-Aguayo, C., Walton, B.L., Sood, A.K. and Berestein-Lopez, G. (2019) Fatty Acid Binding Protein-4 Silencing Attenuates Atherosclerosis Progression by Affecting Macrophage Apoptosis and Autophagy. Journal of Biosciences and Medicines, 7, 99-109.

https://doi.org/10.4236/jbm.2019.77008

Received: April 10, 2019

Accepted: July 12, 2019

Published: July 15, 2019

Copyright $\odot 2019$ by author(s) and Scientific Research Publishing Inc. This work is licensed under the Creative Commons Attribution International License (CC BY 4.0).

http://creativecommons.org/licenses/by/4.0/

\begin{abstract}
Background: Atherosclerosis, which is the principal cause of heart attacks and strokes, is the leading cause of death in the United States. Various biological processes such as apoptosis and autophagy are involved in almost every stage of atherosclerosis, which could lead to plaque instability causing stroke and death. Fatty Acid Binding Protein 4 (FABP4) is an adipokine released by macrophages and is involved in multiple disease conditions including stroke. However, the association of FABP4 with macrophage apoptosis and autophagy in atherosclerosis has not been elucidated. We hypothesize that silencing FABP4 protein could be a novel therapeutic approach to attenuate macrophage apoptosis and autophagy thereby minimizing plaque instability in atherosclerosis. Methods: RAW264 mouse macrophage cells were transfected with siRNA control liposome quantum dots (QD), siFABP4 liposome QD at the concentration of 150 $\mu \mathrm{g} / \mathrm{ml}$ total lipids, or TNF- $\alpha$ at $100 \mathrm{ng} / \mathrm{ml}$. Western blot and reverse phase protein array (RPPA) analysis were completed. Results: Inhibiting the translation of FABP4 blocked the apoptotic pathway as evidenced from the increased expression of anti-apoptotic BCL-xL and BCL-2 along with reduced expression of
\end{abstract}


BAX and activated Caspase 3 levels. Beclin-1 and LC3-II levels were also reduced with knocking down FABP4 indicating the attenuation of autophagy. Conclusion: Targeting FABP4 protects against macrophage processes associated with the progression of atherosclerosis.

\section{Keywords}

Atherosclerosis, Apoptosis, Liposomes, Macrophages, FABP4

\section{Introduction}

Atherosclerosis is one of the leading causes of death in the world [1] which is characterized by lipid accumulation and inflammation. These two processes differentiate atherosclerosis from other chronic inflammatory disorders. Atherosclerosis is an asymptomatic disease process until the progression of plaque leads to flow reduction accelerating clinical manifestations like myocardial infarction or stroke [2] [3]. Plaque instability is a possible source of major cardiovascular events in atherosclerotic patients. Macrophage apoptosis plays an important role in plaque instability [4]. Therefore, attenuating macrophage apoptosis could be a therapeutic approach to protect from plaque instability in atherosclerotic patients.

Autophagy is another important biological process whose hallmark is the packaging of damaged cytoplasmic proteins or organelles into double-membrane autophagosomes. These packages then fuse with a lysosome to form an autolysosome that then degrades the contents and recycles into the cytosol. This is an evolutionarily conserved process and maintains cellular homeostasis [5]. Previous studies have demonstrated the role of autophagy in various pathological conditions and the association with apoptosis [6].

Circulating biomarkers such as fatty acid binding protein 4 (FABP4) have long been postulated to predict cardiovascular risk, but never definitively proven. These cytoplasmic fatty acid binding proteins (FABPs) are lipid chaperons that facilitate the transport of lipids to specific cellular compartments. FABP4, also known as aP2, is an important contributor to the metabolism, inflammatory, atherosclerosis and cardiomyopathy pathways [7]. Plasma levels of FABP4 have been associated with increased cardiovascular disease mortality in type 2 diabetes mellitus men [8] and independently associated with coronary artery disease [9]. FABP4 is increasingly recognized as an important biomarker whose elevated levels are related to increased risk for cancer, cardiomyopathies, atherosclerosis and insulin resistance [7]. It is produced and released from adipocytes, macrophages and endothelial cells [10]. Previous animal studies have shown the protective effect of FABP4 inhibition from early and advanced atherosclerosis [11] [12] [13].

We developed a lipid nanoparticle delivery system to inhibit FABP4 with a goal of targeting atherosclerosis as well as other diseases such as diabetes and obesity. RNA interference (RNAi) is a molecular approach that has been used to treat cancers and possibly other diseases [14]. RNAi delivers small segments of 
RNA known as short-interfering RNA (siRNA) which helps in silencing the targeted genes in turn downregulating the target protein. We have developed a biocompatible system with low toxicity for the delivery of gene-specific RNAi to achieve gene silencing at atherosclerotic plaques with low toxicity by using nano-liposomes. Liposomes consist of a phospholipid bilayer and are spherical molecules with an aqueous core. Liposomes have been approved by US Food and Drug Administration as drug delivery systems for several cancer drugs and antifungals [15]. However, liposomal siRNA has not been extensively utilized in atherosclerosis research because of the immunoreactivity of cationic formulations [16] and the inability to achieve high tissue concentrations. Atherosclerosis being one of the major causes of deaths in USA and all over the world, needs treatment options other than using stents to rupture the plaque and opening the valves. FABP4 is one of the important proteins which is considered as a biomarker in various diseases including atherosclerosis. There is no clear research on how FABP4 is associated with plaque formation and atherosclerosis. In our research we are focusing to explore the various pathways associated with FABP4 and atherosclerosis.We hypothesize that the injection of liposomal FABP4 siRNA treatment will silence local FABP4 expression in RAW264 mouse macrophage cells and downregulate apoptosis.

\section{Materials and Methods}

\subsection{Liposomal siRNA Preparation}

Both siRNA Control and On Target siFABP4 Smart Pool, containing 4 target sequences, were purchased from Dharmacon (La Fayette, CO). Liposomes were prepared with DMPC (1,2-dimyristoyl-sn-glycero-3-phosphocholine) and DMPG (1,2-dimyristoyl-sn-glycero-3-phosphoglycerol) in a 7:3 molar ratio. siRNA, lipids, and quantum dots (QD) will be mixed in the presence of excess tertiary butanol at a ratio of 1:10:40 (weight [w/w/w] siRNA/lipid/quantum dots). The mixture will be vortexed, frozen in an acetone/dry ice bath, lyophilized, and stored at $-20^{\circ} \mathrm{C}$ until use as previously described [17]. QDs exhibit red fluorescence with a maximum emission wavelength of $665 \mathrm{~nm}$. QD are unique in their characteristics of exhibiting a narrow excitation/emission wavelength, as well as increased fluorescence and photostability, which makes them an ideal tool for many biomedical applications such as therapeutics and diagnostics.

\subsection{Cell Cultures}

RAW264 cells (ATCC), mouse macrophage cells were sub-cultured for serial passage in $25 \mathrm{~cm}^{2}$ flasks with $5 \mathrm{ml}$ DMEM medium (ATCC) containing $5 \%$ fetal bovine serum (FBS), $5.6 \mathrm{mmol} / \mathrm{l}$ glucose, $2 \mathrm{mmol} / \mathrm{l} \mathrm{L}$-glutamine, $100 \mathrm{U} / \mathrm{ml}$ penicillin and $100 \mu \mathrm{g} / \mathrm{ml}$ streptomycin.

\subsection{SiRNA Transfection}

Cells were seeded in 6-well-plates and transfection was performed with liposom- 
al siRNA control or liposomal siFABP4 at a final concentration of $150 \mu \mathrm{g} / \mathrm{ml}$ total lipids in serum-free incubation medium. In the tumor necrosis factor- $\alpha$ (TNF- $\alpha$ ) treatment experiment, the cells were incubated with TNF- $\alpha$ (R \& D System, $100 \mathrm{ng} / \mathrm{ml}$ (Minneapolis, MN)) for 3 hours and, then washed and incubated with liposomes. The protein lysates were prepared and western blot, RPPA analyses were performed.

\subsection{Reverse Phase Protein Array (RPPA) Analysis}

Expression of FABP4-regulated proteins involved in atherosclerosis-related signaling networks was examined by conducting the RPPA analysis in RPPA Core Facility at University of Texas M.D. Anderson Cancer Center. This technique is used to perform protein assays on thousands of samples simultaneously. It helps to measure protein expression and protein modifications. Protein lysates were prepared and probed with 172 RPPA-platform-validated antibodies. Primary antibodies will be visualized by $\mathrm{DAB}$ colorimetric reaction and images produced via flatbed scanner. The density of protein expression will be quantified by Array-Pro analyzer. To confirm the RPPA data, we performed Western blotting analysis.

\subsection{Western Blot Analysis}

Protein samples were extracted from the cell lysates of RAW264 cells following control siRNA or FABP4 siRNA treatment. Protein concentration was determined and samples were separated by SDS-PAGE. PVDF membrane was used to transfer the proteins and blocked with 5\% nonfat dry milk. The membranes were incubated with respective primary antibodies (BCL-xL BCL-2, FABP4, Beclin-1, LC3-I, LC3-II, $\beta$-actin, GAPDH) overnight at $4^{\circ} \mathrm{C}$ followed by washes with TBST and incubated with appropriate secondary antibody at room temperature for 2 hours.

\section{Results}

\subsection{Liposomal Uptake into the Macrophages}

Mouse macrophage cell line-RAW264 cells when incubated with control and FABP4 siRNA-QD; the siRNA was taken up into the cells (Figure 1(A)). This was supported by the expression of FABP4 protein in control siRNA whereas it is significantly downregulated in treated cells (Figure 1(B)).

\subsection{Anti-Apoptotic Effect of FABP4siRNA Liposomes}

Apoptosis is a major factor contributing to plaque instability and sudden coronary death. When apoptosis occurs in the thin fibrous cap of advanced lesions, plaques are prone to rupture, which triggers thrombosis and myocardial infarction [4]. We demonstrated that the downregulation of FABP4 induced a significant increase in the expression of anti-apoptotic related proteins, suggesting that reduced apoptosis may be a mechanism underlying the benefits of FABP4 downregulation 
(A)
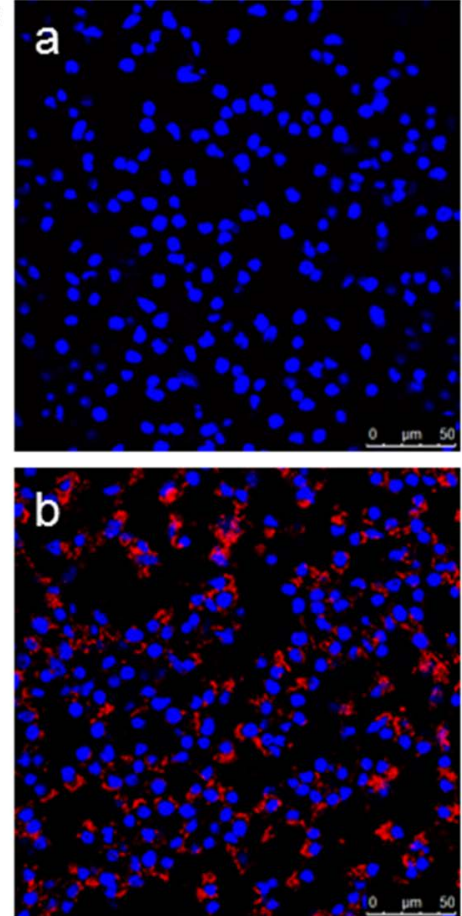

(B)

siControl siFABP4

$\mathrm{Bcl}-\mathrm{xL}(30 \mathrm{kDa})$
$\mathrm{Bcl}-2(26 \mathrm{kDa})$

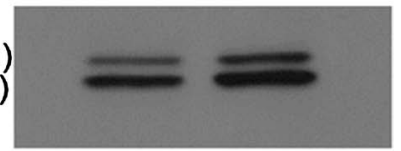

FABP4

$(15 \mathrm{KDa})$

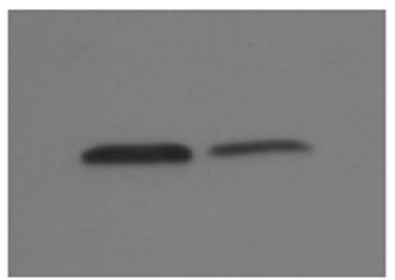

$\beta$-actin

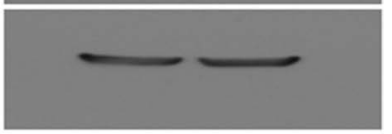

Figure 1. Uptake of liposomal siRNA-QD into RAW264 cells (A). Cells were seeded in 6-well plates, and transfection was performed with liposomal control siRNA-QD (a) or liposomal FABP4 siRNA-QD (b) at a final concentration of $150 \mu \mathrm{g} / \mathrm{ml}$ total lipids in serum-free incubation medium for 1 hour. Confocal microscopy analysis $(63 \times$ magnification) showing liposomal siControl-QD were significantly taken up into the cells. (B) siRNA-mediated knockdown of FABP4 in RAW264 cells (B). Western blot (WB) analysis showed that expression of $F A B P 4$ was greatly reduced in si $F A B P 4$ treated cells. Western blot analysis of anti-apoptotic proteins in control or FABP4 siRNA-treated RAW264 cells is shown (B).

(Figure 1, Figure 2, and Figure 3). Anti-apoptotic proteins Bcl-xL and Bcl-2 were upregulated with FABP4 silencing which is evident from both western blot (Figure 1(B)) and RPPA analysis (Figure 2) whereas expression of pro-apoptotic $\mathrm{BAX}$, which effects the outer membrane permeabilization of mitochondria and the release of cytochrome $\mathrm{c}$ was significantly inhibited (Figure 2).

\subsection{FABP4 siRNA Liposomes Attenuated Apoptosis during TNF- $\alpha$ Stimulation}

Cells were stimulated with TNF- $\alpha$ which is an inflammatory cytokine, to investigate its effect on apoptosis while simultaneously silencing FABP4. TNF- $\alpha$ stimulation increased FABP4 expression and apoptosis which is observed from enhanced caspase 3 activation (Figure 3). However, knocking down FABP4 with siRNA liposomes in these cells downregulated both FABP4 and apoptotic caspase 3 activity (Figure 3 ).

\subsection{Effect of FABP4 Silencing on Autophagy}

Autophagy plays an important role at every stage of atherosclerosis [18], hence 


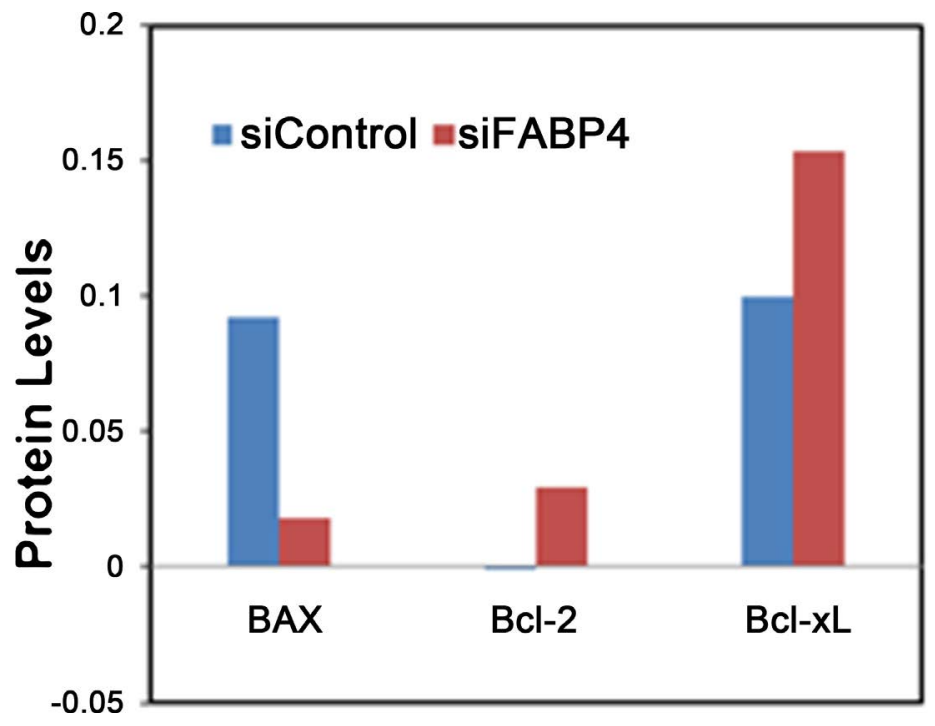

Figure 2. Profile of the FABP4-regulated proteins: Protein lysates were prepared from the RAW264 cells treated with FABP4 or control siRNA and probed with RPPA platform-validated antibodies. Proteins were visualized by using DAB. Assay was run in duplicate. A 16-bit TIFF image was generated, and the density of protein expression was quantified by using an Array-Pro analyzer. The median value is shown.

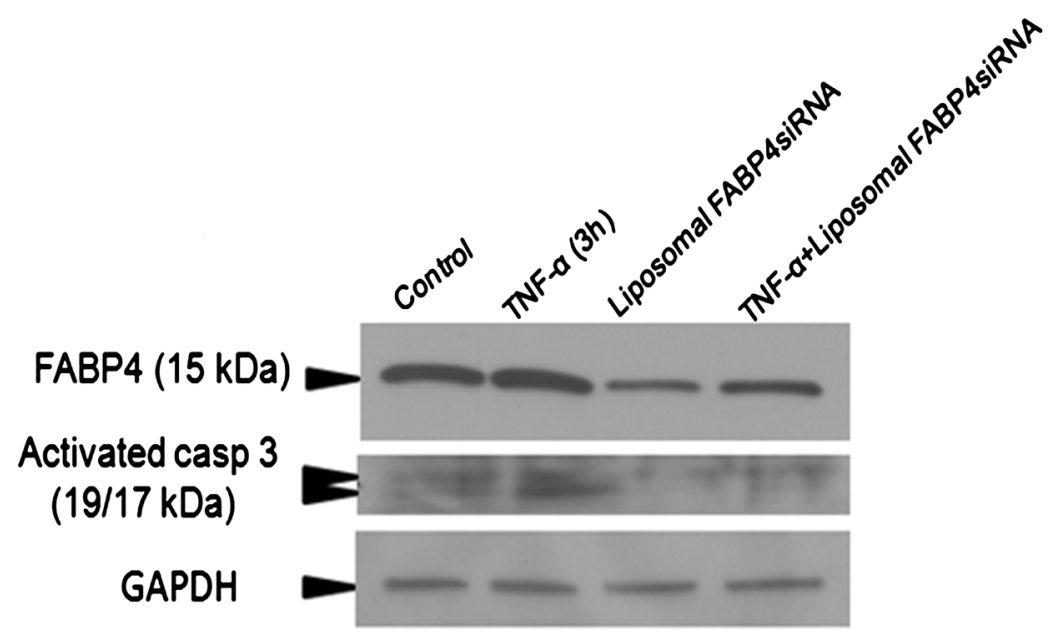

Figure 3. FABP4 overexpression by TNF- $\alpha$ stimulation and reduction of activated caspase-3 expressions by liposomal FABP4 siRNA in RAW264 cells. RAW264 cells were treated with TNF- $\alpha$ for $3 \mathrm{~h}$. Expression of $F A B P 4$ was significantly up-regulated by TNF- $\alpha$ and liposomal FABP4 siRNA treatment induced a significant reduction of FABP4 expression both in TNF- $\alpha$ treated and non-treated groups. Overexpression of FABP4 induced cleavage and activation of pro-caspase 3 to its corresponding active forms. GAPDH was used to as a loading control.

we looked at autophagy markers LC3-I, LC3-II and Beclin-1 to investigate the association between FABP4 and autophagy. Attenuation of autophagic markers was observed in cells silenced from FABP4 using liposomes (Figure 4). Reduced expression of LC3-I, LC3-II and Beclin-1 indicates the effect of FABP4 silencing at autophagosome formation and autolysosome formation which might result in lower degradation of cells. 


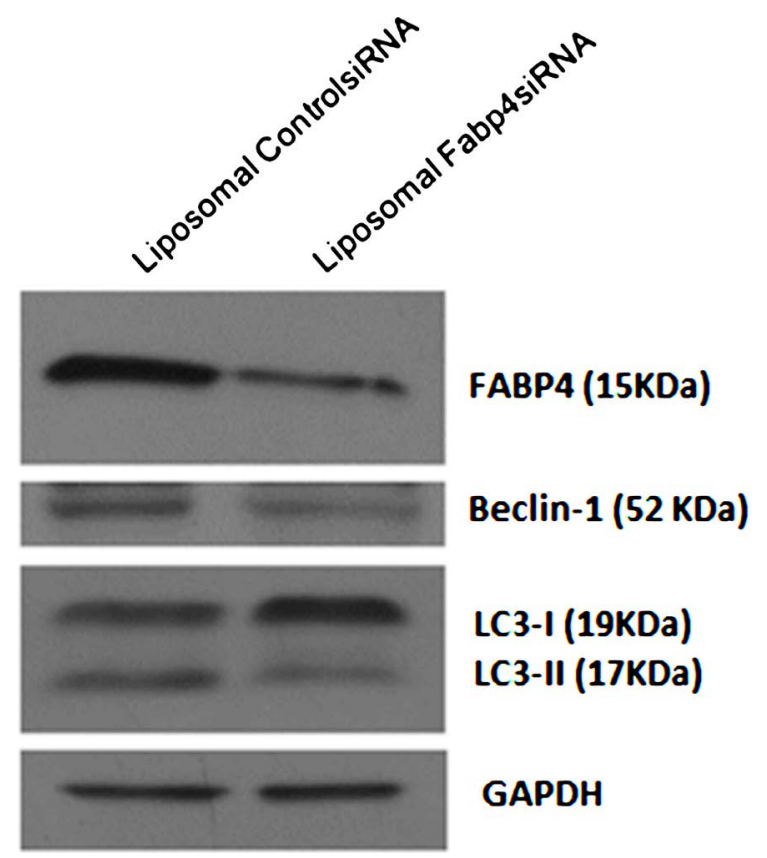

Figure 4. Liposomal FABP4 siRNA inhibits the expressions of Autophagy-associated genes, Beclin-1 and LC3-II in RAW264 cells. Cells were seeded in 6-well plates, and transfection was performed with liposomal siControl or liposomal siFABP4 at a final concentration of $150 \mu \mathrm{g} / \mathrm{ml}$ total lipids in serum-free incubation medium for 48 hours. Western blot analysis showed that the downregulation of FABP4 significantly inhibited the expressions of Autophagy-associated Beclin-1 and LC3-II genes along with FABP4.

\section{Discussion}

Atherosclerosis is the leading cause of death in the world which involves various cellular and molecular changes [19]. Macrophages are gaining attention in the treatment of atherosclerosis for their contribution in necrotic core formation and fibrous cap thinning [20] [21]. Macrophages produce a adipokine-FABP4 which is involved in various processes like lipid metabolism, glucose metabolism, and insulin resistance [10]. Serum levels of FABP4 were reported to be a potential prognostic marker of mortality in patients with stroke [22]. The association between FABP4 and apoptosis was demonstrated earlier [23] [24]. Apoptosis of macrophages with lipid content promotes atherosclerotic plaque instability, rupture and thrombosis due to inflammatory response and necrosis [4]. Macrophages are also accumulated in vulnerable plaques where autophagy plays prominent role to maintain homeostasis [21] [25].

We have previously demonstrated the uptake of anionic liposomes into the atheromas of Watanabe heritable hyperlipidemic rabbits within lipid pools, suggesting that anionic liposome uptake is associated with areas of metabolically active plaque [17]. Recently, we have shown that the uptake of anionic liposomes into plaques in the aortic tissue of ApoE-/- mice and that these liposomes colocalize with and accumulate in macrophages [26]. The current study demonstrated that the uptake of liposomal siRNA QD into macrophage cells was significant (Figure 1). We also observed that silencing FABP4 in mouse macrophages down- 
regulated apoptosis by stimulating anti-apoptotic BCL-2 and BCL-xL while reducing BAX (Figure 1, Figure 2) suggesting that reduced apoptosis may be a mechanism underlying the benefits of FABP4 downregulation. The role of FABP4 in inflammatory conditions was observed by stimulating the mouse macrophage cells with TNF- $\alpha$ which is an inflammatory cytokine. TNF- $\alpha$ stimulated cells have elevated levels of apoptotic proteins (activated caspase-3) whereas cells treated with FABP4siRNA liposomes has inhibited the apoptotic effect.

Previous study has reported that plaque necrosis in advanced atherosclerosis might be due to the defects in clearing apoptotic macrophage cells which results in atherosclerotic cardiovascular events [27]. Autophagy is another biologically conservative process that plays an important role in maintaining lipid metabolism and apoptosis in obesity and atherosclerosis. Alteration of autophagy was observed in atherosclerotic plaques [28]. In present study, we observed attenuation of autophagy when FABP4 is downregulated with siRNA liposomes in macrophages. This is evident from the reduced expression of LC3-II which is a marker for the initiation of autophagy process. Reduction in Beclin-1 levels, marker for activation of autophagosome indicates the decreased formation of autophagosomes attenuating macrophage autophagy (Figure 4).

The fate of macrophage may lie between activating pro-survival mechanism of autophagy and apoptosis as a cell death response [29] [30]. Elevated levels of apoptosis were observed in mice with autophagy deficient macrophages [31]. However, detailed pathways indicating the involvement and association of apoptosis and autophagy with FABP4 in atherosclerotic conditions needs to be elucidated. Thus, targeting circulating inflammatory biomarkers may provide valuable information about the progression of atherosclerosis and helps to avoid adverse events in the near future.

\section{Conclusion}

Previous studies have investigated the small molecule inhibitors but no studies have examined the inhibition of FABP4 using siRNA. In the current study we investigated the association of FABP4 with various biological processes like apoptosis and autophagy. We attenuated apoptosis and autophagy by silencing FABP4 in macrophages which could be an effective therapeutic approach for the prevention and treatment of atherosclerosis. This is the first time that the research on autophagy, apoptosis and atherosclerosis is interconnected in macrophage cell line.

\section{Funding}

This work was supported by the Gillson Longenbaugh Foundation (9091-001-N-1 \& G 2015).

\section{Conflicts of Interest}

The authors declare no conflicts of interest regarding the publication of this paper. 


\section{References}

[1] Weber, C. and Noels, H. (2011) Atherosclerosis: Current Pathogenesis and Therapeutic Options. Nature Medicine, 17, 1410-1422. https://doi.org/10.1038/nm.2538

[2] Hansson, G.K. (2005) Inflammation, Atherosclerosis, and Coronary Artery Disease. The New England Journal of Medicine, 352, 1685-1695.

https://doi.org/10.1056/NEJMra043430

[3] Halvorsen, B., Otterdal, K., Dahl, T.B., Skjelland, M., Gullestad, L., et al. (2008) Atherosclerotic Plaque Stability-What Determines the Fate of a Plaque? Progress in Cardiovascular Diseases, 51, 183-194. https://doi.org/10.1016/j.pcad.2008.09.001

[4] Tabas, I. (2009) Macrophage Apoptosis in Atherosclerosis: Consequences on Plaque Progression and the Role of Endoplasmic Reticulum Stress. Antioxidants \& Redox Signaling, 11, 2333-2339. https://doi.org/10.1089/ars.2009.2469

[5] Rubinsztein, D.C., Marino, G. and Kroemer, G. (2011) Autophagy and Aging. Cell, 146, 682-695. https://doi.org/10.1016/j.cell.2011.07.030

[6] Sergin, I., Evans, T.D., Zhang, X.Y., Bhattacharya, S., et al. (2017) Exploiting Macrophage Autophagy-Lysosomal Biogenesis as a Therapy for Atherosclerosis. $\mathrm{Na}$ ture Communications, 8, Article No. 15750. https://doi.org/10.1038/ncomms 15750

[7] Djousse, L. and Gaziano, J.M. (2012) Plasma Levels of FABP4, But Not FABP3, Are Associated with Increased Risk of Diabetes. Lipids, 47, 757-762. https://doi.org/10.1007/s11745-012-3689-7

[8] Liu, G., Ding, M., Chiuve, S.E., Rimm, E.B., Franks, P.W., et al. (2016) Plasma Levels of Fatty Acid-Binding Protein 4, Retinol-Binding Protein 4, High-Molecular-Weight Adiponectin, and Cardiovascular Mortality among Men with Type 2 Diabetes: A 22-Year Prospective Study. Arteriosclerosis, Thrombosis, and Vascular Biology, 36, 2259-2267. https://doi.org/10.1161/ATVBAHA.116.308320

[9] Doi, M., Miyoshi, T., Hirohata, S., Nakamura, K., Usui, S., et al. (2011) Association of Increased Plasma Adipocyte Fatty Acid-Binding Protein with Coronary Artery Disease in Non-Elderly Men. Cardiovascular Diabetology, 10, 44. https://doi.org/10.1186/1475-2840-10-44

[10] Kralisch, S. and Fasshauer, M. (2013) Adipocyte Fatty Acid Binding Protein: A Novel Adipokine Involved in the Pathogenesis of Metabolic and Vascular Disease? Diabetologia, 56, 10-21. https://doi.org/10.1007/s00125-012-2737-4

[11] Makowski, L., Boord, J.B., Maeda, K., Babaev, V.R., Uysal, K.T., et al. (2001) Lack of Macrophage Fatty-Acid-Binding Protein aP2 Protects Mice Deficient in Apolipoprotein E against Atherosclerosis. Nature Medicine, 7, 699-705. https://doi.org/10.1038/89076

[12] Boord, J.B., Maeda, K., Makowski, L., Babaev, V.R., Fazio, S., et al. (2002) Adipocyte Fatty Acid-Binding Protein, aP2, Alters Late Atherosclerotic Lesion Formation in Severe Hypercholesterolemia. Arteriosclerosis, Thrombosis, and Vascular Biology, 22, 1686-1691. https://doi.org/10.1161/01.ATV.0000033090.81345.E6

[13] Furuhashi, M., Tuncman, G., Görgün, C.Z., Makowski, L., Atsumi, G., et al. (2007) Treatment of Diabetes and Atherosclerosis by Inhibiting Fatty-Acid-Binding Protein aP2. Nature, 447, 959-965. https://doi.org/10.1038/nature05844

[14] Kim, D. and Rossi, J. (2008) RNAi Mechanisms and Applications. Biotechniques, 44, 613-616. https://doi.org/10.2144/000112792

[15] Bulbake, U., Doppalapudi, S., Kommineni, N. and Khan, W. (2017) Liposomal For- 
mulations in Clinical Use: An Updated Review. Pharmaceutics, 9, pii: E12. https://doi.org/10.3390/pharmaceutics9020012

[16] Sakurai, H., Kawabata, K., Sakurai, F., Nakagawa, S. and Mizuguchi, H. (2008) Innate Immune Response Induced by Gene Delivery Vectors. International Journal of Pharmaceutics, 354, 9-15. https://doi.org/10.1016/j.ijpharm.2007.06.012

[17] Walton, B.L., Leja, M., Vickers, K.C., Estevez-Fernandez, M., Sanguino, A., Wang, E., et al. (2010) Delivery of Negatively Charged Liposomes into the Atheromas of Watanabe Heritable Hyperlipidemic Rabbits. Vascular Medicine, 15, 307-313. https://doi.org/10.1177/1358863X10374118

[18] Martinet, W. and De Meyer, G.R. (2008) Autophagy in Atherosclerosis. Current Atherosclerosis Reports, 10, 216-223. https://doi.org/10.1007/s11883-008-0034-y

[19] Libby, P., Ridker, P.M. and Hansson, G.K. (2011) Progress and Challenges in Translating the Biology of Atherosclerosis. Nature, 473, 317-325. https://doi.org/10.1038/nature10146

[20] Moore, K.J., Sheedy, F.J. and Fisher, E.A. (2013) Macrophages in Atherosclerosis: A Dynamic Balance. Nature Reviews Immunology, 13, 709-721. https://doi.org/10.1038/nri3520

[21] Moore, K.J. and Tabas, I. (2011) Macrophages in the Pathogenesis of Atherosclerosis. Cell, 145, 341-355. https://doi.org/10.1016/j.cell.2011.04.005

[22] Tu, W.J., Zeng, X.-W., Deng, A.J., Zhao, S.-J., Luo, D.-Z., et al. (2017) Circulating FABP4 (Fatty Acid-Binding Protein 4) Is a Novel Prognostic Biomarker in Patients with Acute Ischemic Stroke. Stroke, 48, 1531-1538. https://doi.org/10.1161/STROKEAHA.117.017128

[23] Erbay, E., Babaev, V.R., Mayers, J.R., Makowski, L., Charles, K.N., et al. (2009) Reducing Endoplasmic Reticulum Stress through a Macrophage Lipid Chaperone Alleviates Atherosclerosis. Nature Medicine, 15, 1383-1391. https://doi.org/10.1038/nm.2067

[24] Yao, F., Li, Z., Eharad, T., Yang, L., Wang, D.D., et al. (2015) Fatty Acid-Binding Protein 4 Mediates Apoptosis via Endoplasmic Reticulum Stress in Mesangial Cells of Diabetic Nephropathy. Molecular and Cellular Endocrinology, 411, 232-242. https://doi.org/10.1016/j.mce.2015.05.003

[25] Murray, P.J. and Wynn, T.A. (2011) Protective and Pathogenic Functions of Macrophage Subsets. Nature Reviews Immunology, 11, 723-737. https://doi.org/10.1038/nri3073

[26] Zhaorigetu, S., Rodriguez-Aguayo, C., Sood, A.K., Lopez-Berestein, G. and Walton, B.L. (2014) Delivery of Negatively Charged Liposomes into the Atherosclerotic Plaque of Apolipoprotein E-Deficient Mouse Aortic Tissue. Journal of Liposome Research, 24, 182-190. https://doi.org/10.3109/08982104.2013.863208

[27] Zhu, Y.N., Fan, W.-J., Zhang, C., Guo, F., Li, W., et al. (2017) Role of Autophagy in Advanced Atherosclerosis (Review). Molecular Medicine Reports, 15, 2903-2908. https://doi.org/10.3892/mmr.2017.6403

[28] Sergin, I. and Razani, B. (2014) Self-Eating in the Plaque: What Macrophage Autophagy Reveals about Atherosclerosis. Trends in Endocrinology and Metabolism, 25, 225-234. https://doi.org/10.1016/j.tem.2014.03.010

[29] Gump, J.M. and Thorburn, A. (2011) Autophagy and Apoptosis: What Is the Connection? Trends in Cell Biology, 21, 387-392. https://doi.org/10.1016/j.tcb.2011.03.007

[30] Eisenberg-Lerner, A., Bialik, S., Simon, H.-U. and Kimchi, A. (2009) Life and Death 
Partners: Apoptosis, Autophagy and the Cross-Talk between Them. Cell Death \& Differentiation, 16, 966-975. https://doi.org/10.1038/cdd.2009.33

[31] Liao, X., Sluimer, J.C., Wang, Y., Subramanian, M., Brown, K., et al. (2012) Macrophage Autophagy Plays a Protective Role in Advanced Atherosclerosis. Cell Metabolism, 15, 545-553. https://doi.org/10.1016/j.cmet.2012.01.022 